Siberian Mathematical Journal, Vol. 44, No. 6, pp. 1027-1038, 2003

Original Russian Text Copyright (c) 2003 Maximenko E. A.

\title{
CONVOLUTION OPERATORS ON EXPANDING POLYHEDRA: LIMITS OF THE NORMS OF INVERSE OPERATORS AND PSEUDOSPECTRA
}

\section{E. A. Maximenko}

UDC 517.983.34

\begin{abstract}
We consider matrix convolution operators with integrable kernels on expanding polyhedra. We study their connection with convolution operators on the cones at the vertices of polyhedra. We prove that the norm of the inverse operator on a polyhedron tends to the maximum of the norms of the inverse operators on the cones, and the pseudospectrum tends to the union of the corresponding pseudospectra. The study bases on the local method adapted to this kind of problems.
\end{abstract}

Keywords: convolution operator, polyhedron, norm of inverse operator, pseudospectrum

\section{Introduction}

Throughout this article, we fix natural numbers $n$ and $m$ and a number $p \geq 1$.

If $X$ is a measurable subset of $\mathbb{R}^{n}$ then we denote by $L_{p}^{m}(X)$ the product of $m$ copies of the space $L_{p}(X)$ or, which is equivalent, the space of (classes of) vector-functions on $X$ with values in $\mathbb{C}^{m}$ whose $p$ th powers are absolutely integrable. The norm in $L_{p}^{m}(X)$ is defined by the formula

$$
\|f\|_{p}=\left\|\left(f_{1}, \ldots, f_{m}\right)\right\|_{p}=\left(\sum_{j=1}^{m} \int_{X}\left|f_{j}(x)\right|^{p} d x\right)^{1 / p} .
$$

We denote by $\mathbb{C}^{m \times m}$ the space of square matrices of order $m$.

An operator $A$ acting in $L_{p}^{m}\left(\mathbb{R}^{n}\right)$ by the rule

$$
(A f)(y)=c f(y)+\int_{\mathbb{R}^{n}} k(y-x) f(x) d x, \quad y \in \mathbb{R}^{n}, f \in L_{p}^{m}\left(\mathbb{R}^{n}\right),
$$

where $c \in \mathbb{C}^{m \times m}$ and $k$ is an integrable vector-function with values in $\mathbb{C}^{m \times m}$, is called a matrix convolution operator with integrable kernel (or, in other words, with Wiener symbol).

If $A$ is an operator in $L_{p}^{m}\left(\mathbb{R}^{n}\right)$ and $X$ is a measurable subset of $\mathbb{R}^{n}$ then we denote by $A_{X}$ the operator that acts in $L_{p}^{m}(X)$ by the formula

$$
A_{X}=Q_{X} A J_{X}
$$

where the operator $J_{X}: L_{p}^{m}(X) \rightarrow L_{p}^{m}\left(\mathbb{R}^{n}\right)$ extends a vector-function outside $X$ by zero and the operator $Q_{X}: L_{p}^{m}\left(\mathbb{R}^{n}\right) \rightarrow L_{p}^{m}(X)$ restricts the domain of a vector-function.

Suppose that $M$ is a polyhedron in $\mathbb{R}^{n}$ (here and in the sequel, all polyhedra are supposed to be convex) and $E$ is the set of its vertices. With each point $x \in E$ we associate the cone $K_{x}$ with vertex 0 which is generated by the set $M-x$ :

$$
K_{x}=\{\alpha(y-x): \alpha>0, y \in M\} .
$$

In this article we consider some Banach algebra $\mathscr{W}_{M}$ generated by convolution operators on the family of sets $\{\tau M\}_{\tau>0}$. Given a point $x \in M$, we introduce the "local equivalence" relation $\stackrel{x}{\sim}$ and construct

Rostov-on-Don. Translated from Sibirskiŭ Matematicheskiŭ Zhurnal, Vol. 44, No. 6, pp. 1310-1323, November-December, 2003. Original article submitted May 14, 2003. 
an isomorphism of the corresponding quotient algebra $\left(\mathscr{W}_{M}\right)_{x}$ onto the algebra $\mathscr{C}_{K_{x}}$ generated by the convolution operators on the cone $K_{x}$. We then define a morphism of $\mathscr{W}_{M}$ to the product of the family of the algebras $\left\{\mathscr{C}_{K_{x}}\right\}_{x \in E}$. The main result of this article is Theorem 6.1 claiming that this "global morphism" is an isometry and "agrees with inversion."

We now present only two assertions that result from Theorem 6.1: on the limits of the norms of inverse operators and on the limits of pseudospectra. Given a Banach algebra $\mathscr{A}$ with unity $e$ and norm $|\cdot|, a \in \mathscr{A}$, and $\varepsilon>0$, the $\varepsilon$-pseudospectrum of $a$ is the set

$$
\sigma_{\varepsilon}(a)=\left\{\lambda \in \mathbb{C}||(\lambda e-a)^{-1} \mid \geq 1 / \varepsilon\right\} .
$$

(Here and in the sequel, given a noninvertible element $b$ we put $\left|b^{-1}\right|=+\infty$.)

Proposition 0.1. Let $A$ be a matrix convolution operator in $L_{p}^{m}\left(\mathbb{R}^{n}\right)$. Then

$$
\lim _{\tau \rightarrow+\infty}\left\|A_{\tau M}^{-1}\right\|=\max _{x \in E}\left\|A_{K_{x}}^{-1}\right\| .
$$

Proposition 0.2. Suppose that $A$ is a matrix convolution operator in $L_{p}^{m}\left(\mathbb{R}^{n}\right)$, with $p>1$, and $\varepsilon>0$. Then

$$
\lim _{\tau \rightarrow+\infty} \sigma_{\varepsilon}\left(A_{\tau M}\right)=\bigcup_{x \in E} \sigma_{\varepsilon}\left(A_{K_{x}}\right),
$$

where the convergence of sets is understood in the sense of the Hausdorff metric.

The study of pseudospectra of Toeplitz matrices (in the scalar case and for smooth symbols) originated with the articles of H. Landau [1] and L. Reichel and L. N. Trefethen [2]. Results for the block Toeplitz matrices and matrix Wiener-Hopf operators were obtained by A. Böttcher [3]. Using the methods of $C^{*}$-algebras, he considered only the case of $L_{2}$ but for a very broad class of symbols which contained all piecewise continuous functions. Later, A. Böttcher and H. Wolf [4] studied Toeplitz operators on $n$-dimensional cubes and established some analogs of Propositions 0.1 and 0.2 for them. S. M. Grudsky and A. V. Kozak [5] proved Proposition 0.1 for scalar Toeplitz operators in $L_{1}$ by straightforward computations. A. Böttcher, S. M. Grudsky, and B. Silbermann [6] proved Propositions 0.1 and 0.2 for one-dimensional matrix convolutions with integrable kernels in $L_{p}$. The history of this problem is exposed in more detail in [6] and in A. Böttcher and B. Silbermann's monograph [7, Chapter 3 and $\S 6.3$ ].

In this article we generalize the results of [6] to the multidimensional case. We borrow the idea of Theorem 6.1 and the methods of dealing with pseudospectra from [6]. On the other hand, the proof of Theorem 6.1 bases on the local method whose prototype (called also the theory of local-type operators) was developed by I. B. Simonenko [8-12] while studying the Noether property of singular integral operators. A. V. Kozak [13-15] generalized this method to abstract Banach algebras furnished with a local structure and applied it to studying the invertibility of convolution operators on expanding subsets of $\mathbb{R}^{n}$ (see also [16]). We employ the local method in A. V. Kozak's interpretation, supplementing it with N. Ya. Krupnik's theorem on the norm of a local-type operator [17].

\section{$\S 1$. Pure Subalgebras}

In this section we give elementary facts about pure subalgebras and morphisms agreeing with inversion.

Henceforth by a Banach algebra we mean a unital Banach algebra (the unit is denoted by $e$ ); by a morphism of Banach algebras we mean a morphism of unital Banach algebras; and subalgebras are not supposed to be closed. We use the symbol $\operatorname{Inv}(\mathscr{A})$, where $\mathscr{A}$ is a Banach algebra, to denote the set of invertible elements of $\mathscr{A} ; \sigma(a)$, with $a \in \mathscr{A}$, is the spectrum of $a$.

A subalgebra $\mathscr{B}$ of an algebra $\mathscr{A}$ is pure if the unit of $\mathscr{A}$ belongs to $\mathscr{B}$ and $\operatorname{Inv}(\mathscr{B})=\mathscr{B} \cap \operatorname{Inv}(\mathscr{A})$. If $X \subset \mathscr{A}$ then $[X]$ stands for the closed pure subalgebra of $\mathscr{A}$ generated by $X$. Since the closure of a pure subalgebra is a pure subalgebra, $[X]$ is the closure of the pure subalgebra generated by $X$.

Let $f: \mathscr{A} \rightarrow \mathscr{B}$ be a morphism of Banach algebras. It follows from $a \in \operatorname{Inv}(\mathscr{A})$ that $f(a) \in \operatorname{Inv}(\mathscr{B})$ and $f(a)^{-1}=f\left(a^{-1}\right)$. We say that the morphism $f$ agrees with inversion if the conditions $a \in \operatorname{Inv}(\mathscr{A})$ and $f(a) \in \operatorname{Inv}(\mathscr{B})$ are equivalent for every $a \in \mathscr{A}$. 
Proposition 1.1. Suppose that $f: \mathscr{A} \rightarrow \mathscr{B}$ is an isometric morphism of Banach algebras which agrees with inversion and $X \subset \mathscr{A}$. Then $f([X])=[f(X)]$.

Proof. Let $Y_{1}$ and $Y_{2}$ be the pure subalgebras of $\mathscr{A}$ and $\mathscr{B}$ generated by $X$ and $f(X)$. Since $f$ agrees with inversion, $f\left(Y_{1}\right)=Y_{2}$. Since $f$ is an isometry of complete spaces, $f\left(\bar{Y}_{1}\right)=\overline{f\left(Y_{1}\right)}=\bar{Y}_{2}$, where the bar denotes the closure. However, $f\left(\bar{Y}_{1}\right)=f([X])$ and $\bar{Y}_{2}=[f(X)]$. Thus, $f([X])=[f(X)]$.

For the convenience of reference, we give an obvious assertion on uniqueness of extension of a morphism to a pure subalgebra.

If $f: X \rightarrow Y$ is a mapping and $X_{1} \subset X$ then we denote the restriction of $f$ to $X_{1}$ by $f \mid X_{1}$.

Proposition 1.2. Suppose that $\mathscr{A}$ and $\mathscr{B}$ are Banach algebras, $X \subset \mathscr{A}$, and $f:[X] \rightarrow \mathscr{B}$ and $g:[X] \rightarrow \mathscr{B}$ are morphisms of Banach algebras such that $f|X=g| X$. Then $f=g$.

\section{$\S$ 2. The Local Method}

In this section we give a version of the local method (or "local principle"), namely the local method in A. V. Kozak's interpretation [13,15]. We replace the condition (LS4') of Kozak's articles with the stronger condition (LS4) introduced by N. Ya. Krupnik [17] to prove Theorem 2.3.

First of all, we agree on the topological terms and notations. If $\mathscr{X}$ is a topological space then $\Sigma_{\mathscr{X}}$ is the ring of Borel subsets of $\mathscr{X}$; a neighborhood of a point $x(x \in \mathscr{X})$ is an open subset of $\mathscr{X}$ containing $x$; $\mathfrak{U}_{x}(x \in \mathscr{X})$ is the set of neighborhoods of $x$; and $\bar{u}(u \subset \mathscr{X})$ is the closure of $u$.

We say that $(\mathscr{A}, \mathscr{X}, p)$ is an algebra with local structure if $\mathscr{A}$ is a Banach algebra (with norm $|\cdot|$ and unit $e$ ), $\mathscr{X}$ is a compact set, $p: \Sigma_{\mathscr{X}} \rightarrow \mathscr{A}$, and the following properties are satisfied:

(LS1) $p(\mathscr{X})=e$;

(LS2) $p(u \cap v)=p(u) p(v)$ for arbitrary $u, v \in \Sigma_{\mathscr{X}}$;

(LS3) $p(u \cup v)=p(u)+p(v)$ for arbitrary $u, v \in \Sigma_{\mathscr{X}}$ such that $\bar{u} \cap \bar{v}=\varnothing$;

(LS4) $|p(u) a p(u)+p(v) b p(v)| \leq \max (|a|,|b|)$ for arbitrary $a, b \in \mathscr{A}$ and $u, v \in \Sigma_{\mathscr{X}}$ such that $u \cap v=\varnothing$.

Below in this section we assume that $(\mathscr{A}, \mathscr{X}, p)$ is an algebra with local structure.

It follows from (LS2) that the elements of the form $p(u)$ are idempotent: $p(u)^{2}=p(u)$. Using (LS4), we find that $|p(u)|=1$ for $p(u) \neq 0$. In particular,

$\left(\mathrm{LS}^{\prime}\right) \sup _{u \in \Sigma_{\mathscr{C}}}|p(u)|<+\infty$.

Let $1_{u}, u \in \Sigma_{\mathscr{X}}$, be the characteristic function of the set $u ; B(\mathscr{X})$, the Banach algebra of bounded functions on $\mathscr{X}$ with the uniform norm; $S(\mathscr{X})$, the closed subalgebra of $B(\mathscr{X})$ generated by the elements of the form $1_{u}\left(u \in \Sigma_{\mathscr{X}}\right)$; and $C(\mathscr{X})$, the Banach algebra of continuous functions on $\mathscr{X}$. It is easy to see that $C(\mathscr{X}) \subset S(\mathscr{X})$.

An element $a \in \mathscr{A}$ is said to be of local type if $p(u) a p(v)=0$ for arbitrary $u, v \in \Sigma_{\mathscr{X}}$ such that $\bar{u} \cap \bar{v}=\varnothing$. We denote the set of elements of local type by $\mathscr{A}^{\prime}$.

Proposition 2.1. The mapping $1_{u} \mapsto p(u)\left(u \in \Sigma_{\mathscr{X}}\right)$ has a unique extension to a morphism $\mu: S(\mathscr{X}) \rightarrow \mathscr{A}$ of Banach algebras. This morphism does not increase the norm: $|\mu(\varphi)| \leq\|\varphi\|$ for all $\varphi \in S(\mathscr{X})$.

Theorem 2.1. $\mathscr{A}^{\prime}$ consists of those and only those elements which commute with the set $\mu(C(\mathscr{X}))$. Thereby $\mathscr{A}^{\prime}$ is a closed pure subalgebra of $\mathscr{A}$.

See the proofs of Proposition 2.1 and Theorem 2.1 in $[13,15]$.

In line with [12], given a point $x \in \mathscr{X}$, we define the following seminorms:

$$
\begin{gathered}
q_{L}(a, x)=\inf _{u \in \mathfrak{U}_{x}}|p(u) a|, \quad q_{R}(a, x)=\inf _{u \in \mathfrak{U}_{x}}|a p(u)|, \\
q(a, x)=\max \left(q_{L}(a, x), q_{R}(a, x)\right) .
\end{gathered}
$$

Clearly, $q(a, x) \leq|a|$ for every $a \in \mathscr{A}$. 
If $a \in \mathscr{A}^{\prime}$ and $x \in \mathscr{X}$ then

$$
q(a, x)=\inf _{u \in \mathfrak{U}_{x}}|p(u) a|=\inf _{u \in \mathfrak{U}_{x}}|\operatorname{ap}(u)|=\inf _{u \in \mathfrak{U}_{x}}|p(u) a p(u)| .
$$

For each point $x \in \mathscr{X}$ the seminorm $q(\cdot, x)$ generates the equivalence relation $\stackrel{x}{\sim}$ :

$$
a \stackrel{x}{\sim} b \Leftrightarrow q(a-b, x)=0 .
$$

We can easily verify the following properties:

(a) if $a \stackrel{x}{\sim} b$ then $q(a, x)=q(b, x)$;

(b) if $a_{1} \stackrel{x}{\sim} b_{1}, a_{2} \stackrel{x}{\sim} b_{2}$, and $\lambda, \mu \in \mathbb{C}$ then $\lambda a_{1}+\mu a_{2} \stackrel{x}{\sim} \lambda b_{1}+\mu b_{2}$;

(c) if $a_{n} \rightarrow a, b_{n} \rightarrow b$, and $a_{n} \stackrel{x}{\sim} b_{n}$ then $a \stackrel{x}{\sim} b$;

(d) if $a_{1}, a_{2}, b_{1}, b_{2} \in \mathscr{A}^{\prime}, a_{1} \stackrel{x}{\sim} b_{1}$, and $a_{2} \stackrel{x}{\sim} b_{2}$ then $a_{1} a_{2} \stackrel{x}{\sim} b_{1} b_{2}$;

(e) if $a, b \in \operatorname{Inv}\left(\mathscr{A}^{\prime}\right)$ and $a \stackrel{x}{\sim} b$ then $a^{-1} \stackrel{x}{\sim} b^{-1}$.

Consider $\stackrel{x}{\sim}$ as an equivalence relation on $\mathscr{A}^{\prime}$. Let $\mathscr{A}_{x}^{\prime}$ be the corresponding quotient set and let $\pi_{x}: \mathscr{A}^{\prime} \rightarrow \mathscr{A}_{x}^{\prime}$ be the corresponding quotient mapping. The properties (a), (b), and (d) show that $\mathscr{A}_{x}^{\prime}$ naturally becomes a Banach algebra with unit $\pi_{x}(e)$ and norm $\left|\pi_{x}(\cdot)\right|=q(\cdot, x)$.

Theorem 2.2 (see $[9,11-13,15]$ ). Suppose that $a \in \mathscr{A}^{\prime}$ and that, for every $x \in \mathscr{X}$, the element $\pi_{x}(a)$ is left (right) invertible in the quotient algebra $\mathscr{A}_{x}^{\prime}$. Then $a$ is left (right) invertible.

Theorem 2.3. If $a \in \mathscr{A}^{\prime}$ then $|a|=\sup _{x \in \mathscr{X}} q(a, x)$.

This theorem was proven in N. Ya. Krupnik's article [17] in some particular case but can be trivially generalized to the case of algebras with local structure.

\section{$\S 3$. The Main Objects: the Algebras $\mathscr{A}_{X}, \mathscr{W}_{X}$, and $\mathscr{C}_{X}$}

We define the main objects of the article and show how they are connected with the objects of $\S 2$.

As mentioned in the introduction, the numbers $n \in \mathbb{N}, m \in \mathbb{N}$, and $p \in[1,+\infty)$ are fixed throughout the article.

Given a point $x \in \mathbb{R}^{n}$, denote by $\mathfrak{U}_{x}$ the set of neighborhoods of $x$ (the neighborhoods are supposed to be open).

For every measurable subset $X$ of $\mathbb{R}^{n}$, define the operator $P_{X}: L_{p}^{m}\left(\mathbb{R}^{n}\right) \rightarrow L_{p}^{m}\left(\mathbb{R}^{n}\right)$ by the following rule:

$$
\left(P_{X} f\right)(x)= \begin{cases}f(x), & x \in X \\ 0, & x \in \mathbb{R}^{n} \backslash X\end{cases}
$$

Clearly, $P_{X}=J_{X} Q_{X}$.

For brevity, below we write $L_{p}^{m}$ rather than $L_{p}^{m}\left(\mathbb{R}^{n}\right)$.

If $\mathscr{B}$ is a Banach space then we denote by $\operatorname{End}(\mathscr{B})$ the Banach algebra of bounded linear operators in $\mathscr{B}$.

Take and fix until the end of this section some measurable subset $X$ of $\mathbb{R}^{n}$ (in the next sections we take $X$ to be a cone or polyhedron).

Note that $P_{X}\left(\operatorname{End}\left(L_{p}^{m}\right)\right) P_{X}$ is a Banach algebra with unit $P_{X}$. For brevity, we denote it by $\operatorname{End}\left(P_{X} L_{p}^{m}\right)$. We identify $P_{X} L_{p}^{m}$ with $L_{p}^{m}(X)$ and $\operatorname{End}\left(P_{X} L_{p}^{m}\right)$ with $\operatorname{End}\left(L_{p}^{m}(X)\right)$. If $A \in \operatorname{End}\left(L_{p}^{m}\right)$ then we identify the operator $A_{X}=Q_{X} A J_{X}$ with the element $P_{X} A P_{X}$ of $\operatorname{End}\left(P_{X} L_{p}^{m}\right)$. If $A \in \operatorname{End}\left(L_{p}^{m}\right)$ and if $P_{X} A P_{X}$ is invertible in the algebra $\operatorname{End}\left(P_{X} L_{p}^{m}\right)$ (which is equivalent to the fact that the operator $Q_{X} A J_{X} \in \operatorname{End}\left(L_{p}^{m}(X)\right)$ is invertible) then we identify the corresponding inverse element with the operator $\left(Q_{X} A J_{X}\right)^{-1}$ and denote it by $A_{X}^{-1}$.

Let $\mathscr{U}_{X}$ be the product of the family $\left\{\operatorname{End}\left(P_{\tau X} L_{p}^{m}\right)\right\}_{\tau>0}$, i.e., the set of families of operators of the form $\left\{A_{\tau}\right\}_{\tau>0}$, where $A_{\tau} \in \operatorname{End}\left(P_{\tau X} L_{p}^{m}\right)$ and $\sup _{\tau>0}\left\|A_{\tau}\right\|<+\infty$, furnished with the coordinatewise operations and the norm

$$
\left|\left\{A_{\tau}\right\}_{\tau>0}\right|_{\mathscr{U}_{X}}=\sup _{\tau>0}\left\|A_{\tau}\right\| .
$$


Consider the closed ideal $\mathscr{J}_{X}$ in $\mathscr{U}_{X}$ :

$$
\mathscr{J}_{X}=\left\{\left\{A_{\tau}\right\}_{\tau>0} \in \mathscr{U}_{X} \mid \lim _{\tau \rightarrow+\infty}\left\|A_{\tau}\right\|=0\right\}
$$

Denote by $\mathscr{A}_{X}$ the quotient algebra $\mathscr{U}_{X} / \mathscr{J}_{X}$. The elements of this quotient algebra have the form $a=\left\{A_{\tau}\right\}_{\tau>0}+\mathscr{J}_{X}$, where $A_{\tau} \in \operatorname{End}\left(P_{X} L_{p}^{m}\right)$ and $\sup _{\tau>0}\left\|A_{\tau}\right\|<+\infty$. The norm in $\mathscr{A}_{X}$ is calculated by the formula

$$
\left|\left\{A_{\tau}\right\}_{\tau>0}+\mathscr{J}_{X}\right|=\limsup _{\tau \rightarrow+\infty}\left\|A_{\tau}\right\| .
$$

Define the mapping $j_{X}: \operatorname{End}\left(L_{p}^{m}\right) \rightarrow \mathscr{A}_{X}$ :

$$
j_{X}(A)=\left\{P_{\tau X} A P_{\tau X}\right\}_{\tau>0}+\mathscr{J}_{X}, \quad A \in \operatorname{End}\left(L_{p}^{m}\right) .
$$

Let $\dot{\mathbb{R}}^{n}=\mathbb{R}^{n} \cup\{\infty\}$ be the compact space obtained by completing $\mathbb{R}^{n}$ with one point at infinity, let $\bar{X}$ be the closure of $X$ in $\dot{\mathbb{R}}^{n}$, and let $\Sigma_{X}$ be the set of measurable subsets of $\bar{X}$. Given a set $u \in \Sigma_{X}$, put

$$
p(u)=\left\{P_{\tau(u \cap X)}\right\}_{\tau>0}+\mathscr{J}_{X} .
$$

Lemma 3.1 (see $[11,17]$ ). Suppose that $A, B \in \operatorname{End}\left(L_{p}^{m}\right)$ and $Y$ and $Z$ are disjoint measurable subsets of $\mathbb{R}^{n}$. Then $\left\|P_{Y} A P_{Y}+P_{Z} B P_{Z}\right\| \leq \max (\|A\|,\|B\|)$.

Proposition 3.1 (see $[13,15]) .\left(\mathscr{A}_{X}, \bar{X}, p\right)$ is an algebra with local structure. If $\varphi \in S(\bar{X})$ then $\mu(\varphi)=\left\{M\left(\varphi_{\tau}\right)\right\}_{\tau>0}+\mathscr{J}_{X}$, where $M\left(\varphi_{\tau}\right)$ is the operator of multiplication by the function $\varphi_{\tau}$ defined by the formula

$$
\varphi_{\tau}(x)=\varphi(x / \tau), \quad x \in \mu X, \tau>0 .
$$

Formally, the only new point in Proposition 3.1 is the fact that the condition (LS4) holds for $\left(\mathscr{A}_{X}, \bar{X}, p\right)$; this follows from Lemma 3.1.

Denote by $W_{p}$ the closure in $\operatorname{End}\left(L_{p}^{m}\right)$ of the set of matrix convolution operators, i.e., operators of the form (0.1), where $c \in \mathbb{C}^{m \times m}$ and $k \in L_{1}^{m \times m}\left(\mathbb{R}^{n}\right)$.

Let $\mathscr{C}_{X}=\left[P_{X} W_{p} P_{X}\right]$ and $\mathscr{W}_{X}=\left[j_{X}\left(W_{p}\right)\right]$. In more detail, $\mathscr{C}_{X}$ is the closed pure subalgebra of the Banach algebra $\operatorname{End}\left(P_{X} L_{p}^{m}\right)$ which is generated by the set $\left\{P_{X} A P_{X} \mid A \in W_{p}\right\}$ and $\mathscr{W}_{X}$ is the closed pure subalgebra of the Banach algebra $\mathscr{A}_{X}$ which is generated by the set $\left\{j_{X}(A) \mid A \in W_{p}\right\}$.

Proposition 3.2. $\mathscr{W}_{X} \subset \mathscr{A}_{X}^{\prime}$.

Proof (see $[10,14,16])$. Let $A$ be a matrix convolution operator with compactly-supported kernel; i.e., $A$ has the form (0.1), where $c \in \mathbb{C}^{m \times m}$ and $k \in L_{1}^{m \times m}$; moreover, $k(x)=0$ for $|x| \geq d, d>0$. Then $P_{Y} A P_{Z}=0$ for $\operatorname{dist}(Y, Z)>d$, where

$$
\operatorname{dist}(Y, Z)=\inf _{y \in Y} \inf _{z \in Z}|y-z|
$$

If $u, v \subset X$ and $\bar{u} \cap \bar{v}=\varnothing$ then $r=\operatorname{dist}(u, v)>0$; moreover, $\operatorname{dist}(\tau u, \tau v)>d$ and $P_{\tau u} A P_{\tau v}=0$ for $\tau>d / r$. Hence, $p(u) j_{X}(A) p(v)=0$.

Thus, $j_{X}(A) \in \mathscr{A}_{X}^{\prime}$ if $A$ has the indicated form. However, the operators of this form constitute a dense subset in $W_{p}$; therefore, $j_{X}\left(W_{p}\right) \subset \mathscr{A}_{X}^{\prime}$. By Theorem 2.1, $\mathscr{A}_{X}^{\prime}$ is a closed pure subalgebra of $\mathscr{A}_{X}$. Hence, $\mathscr{W}_{X}=\left[j_{X}\left(W_{p}\right)\right] \subset \mathscr{A}_{X}^{\prime}$, and the proposition is proven.

Propositions 3.1 and 3.2 enable us to use the notations of $\S 2$ for the algebra $\mathscr{A}_{X}$ (in particular, $q(\cdot, x)$ and $\stackrel{x}{\sim}$, where $x \in \bar{X}$ ) and apply all assertions concerning local-type elements to the elements of $\mathscr{W}_{X}$. Given a point $x \in \bar{X}$, we denote by $\left(\mathscr{W}_{X}\right)_{x}$ the algebra $\pi_{x}\left(\mathscr{W}_{X}\right)=\mathscr{W}_{X} / \stackrel{x}{\sim}$. 


\section{$\S$ 4. The Case of Cones}

Given $X \subset \mathbb{R}^{n}$ and $y \in \mathbb{R}^{n}$, put

$$
\operatorname{con}_{y}(X)=\{\lambda(x-y): \lambda>0, x \in X\} .
$$

A set $K \subset \mathbb{R}^{n}$ is called cone with vertex $y$ if $K=\operatorname{con}_{y}(K)$.

Let $K$ be a measurable cone in $\mathbb{R}^{n}$ with vertex 0 ; we assume it fixed till the end of the section.

Lemma 4.1. Suppose that $A \in \operatorname{End}\left(P_{K} L_{p}\right)$ and

$$
a=j_{K}(A)=\{A\}_{\tau>0}+\mathscr{J}_{K} \text {. }
$$

Then $|a|=q(a, 0)=\|A\|$. If $u$ is some neighborhood of 0 and $a=j_{K}(A)=\left\{B_{\tau}\right\}_{\tau>0}+\mathscr{J}_{K}$ then

$$
A=\operatorname{s-lim}_{\tau \rightarrow+\infty} P_{\tau(u \cap K)} B_{\tau} P_{\tau(u \cap K)}=\operatorname{s-lim}_{\tau \rightarrow+\infty} P_{\tau(u \cap K)} B_{\tau}=\operatorname{s-lim}_{\tau \rightarrow+\infty} B_{\tau} P_{\tau(u \cap K)} .
$$

(Here and in the sequel s-lim denotes the pointwise limit.)

Proof. Formula (4.2) follows from the relations

$$
\lim _{\tau \rightarrow+\infty} B_{\tau}=A, \quad \operatorname{s}_{\tau \rightarrow+\infty} P_{\tau(u \cap K)}=\operatorname{sim}_{\tau \rightarrow+\infty} P_{\tau(u \cap \bar{K})}=P_{K} .
$$

From (4.2) and the properties of pointwise convergence of operators we obtain

$$
\|A\| \leq \liminf _{\tau \rightarrow+\infty}\left\|P_{\tau(u \cap K)} B_{\tau}\right\| \leq|p(u \cap K) a|,
$$

whence $\|A\| \leq q_{L}(a, 0)$. Similarly, $\|A\| \leq q_{R}(a, 0)$. Thus, $\|A\| \leq q(a, 0)$. The inequalities $q(a, 0) \leq|a| \leq$ $\|A\|$ are obvious.

Lemma 4.2. Suppose that $A \in \operatorname{End}\left(P_{K} L_{p}\right), a=j_{K}(A)$, and $a \in \mathscr{A}_{K}^{\prime}$. Then the following conditions are equivalent:

(a) the operator $A$ is invertible on $K$;

(b) the element a of the algebra $\mathscr{A}_{K}$ is invertible;

(c) the element $\pi_{0}(a)$ of the quotient algebra $\left(\mathscr{A}_{K}^{\prime}\right)_{0}$ is invertible.

If these conditions are satisfied then $a^{-1}=\left\{A_{K}^{-1}\right\}_{\tau>0}+\mathscr{J}_{K}$.

A somewhat different version of this assertion was proven in $[13,15]$.

Recall that $\left(\mathscr{W}_{K}\right)_{0}=\left(\mathscr{W}_{K} / \stackrel{0}{\sim}\right)=\pi_{0}\left(\mathscr{W}_{K}\right)$.

Proposition 4.1. The mappings $\left(j_{K} \mid \mathscr{C}_{K}\right): \mathscr{C}_{K} \rightarrow \mathscr{W}_{K}$ and $\left(\pi_{0} \mid \mathscr{W}_{K}\right): \mathscr{W}_{K} \rightarrow\left(\mathscr{W}_{K}\right)_{0}$ are isometric morphisms of Banach algebras, and thereby the mapping isocon ${ }_{K}=\left(j_{K} \mid \mathscr{C}_{K}\right)^{-1} \circ\left(\pi_{0} \mid \mathscr{W}_{K}\right)^{-1}$ is an isometric isomorphism of $\left(\mathscr{W}_{K}\right)_{0}$ onto $\mathscr{C}_{K}$.

Proof. It is easily seen that $j_{K}\left(\mathscr{C}_{K}\right) \subset \mathscr{W}_{K}$. It follows from Lemma 4.1 that the mapping $j_{K}$ : $\operatorname{End}\left(P_{K} L_{p}\right) \rightarrow \mathscr{A}_{K}$ is an isometric morphism of Banach algebras. Lemma 4.2 and Proposition 3.2 show that the morphism $j_{K} \mid \mathscr{C}_{K}$ agrees with inversion. By Proposition 1.1, $j_{K} \mid \mathscr{C}_{K}$ is an isometric isomorphism of $\mathscr{C}_{K}$ onto $\mathscr{W}_{K}$. The assertion concerning the morphism $\pi_{0} \mid \mathscr{W}_{K}$ ensues from Lemmas 4.1 and 4.2.

Proposition 4.2. Suppose that $b \in \mathscr{W}_{K}$ and $b=\left\{B_{\tau}\right\}_{\tau>0}+\mathscr{J}_{K}$. Then

$$
\operatorname{isocon}_{K}\left(\pi_{0}(b)\right)=\operatorname{s-lim}_{\tau \rightarrow+\infty} B_{\tau} .
$$

Proof. Let $A=\operatorname{isocon}_{K}\left(\pi_{0}(b)\right)$ and $f \in P_{K} L_{p}$. We have to demonstrate that $\lim _{\tau}\left\|\left(B_{\tau}-A\right) f\right\|=0$. Put $a=\{A\}_{\tau>0}+\mathscr{J}_{K}$. Then $\pi_{0}(a)=\pi_{0}(b)$. Take an arbitrary $\varepsilon>0$ and find a neighborhood $u$ of the origin such that $|(a-b) p(u)|<\varepsilon$, i.e.,

$$
\limsup _{\tau \rightarrow+\infty}\left\|\left(B_{\tau}-A\right) P_{\tau u}\right\|<\varepsilon
$$

Since $\lim _{\tau}\left\|B_{\tau} P_{\tau u} f-B_{\tau} f\right\|=0$ and $\lim _{\tau}\left\|A P_{\tau u} f-A f\right\|=0$, we obtain

$$
\limsup _{\tau \rightarrow+\infty}\left\|\left(B_{\tau}-A\right) f\right\|=\limsup _{\tau \rightarrow+\infty}\left\|\left(B_{\tau}-A\right) P_{\tau u} f\right\|<\varepsilon .
$$

Once $\varepsilon>0$ is chosen arbitrarily, we have $\lim _{\tau \rightarrow+\infty}\left\|\left(B_{\tau}-A\right) f\right\|=0$. 


\section{$\S 5$. The Local Isomorphism}

This section bases on the ideas of [14] (see also [10]).

Let $T_{h}, h \in \mathbb{R}^{n}$, be the operator of translation by $h$ in $L_{p}^{m}$ :

$$
\left(T_{h} f\right)(y)=f(y-h), \quad y \in \mathbb{R}^{n}, f \in L_{p}^{m},
$$

where $\Sigma$ is the set of measurable subsets of $\mathbb{R}^{n}$. Note that if $X \in \Sigma$ and $h \in \mathbb{R}^{n}$ then $T_{h} P_{X}=P_{X+h} T_{h}$.

Suppose that $X, Y \subset \mathbb{R}^{n}, x \in \bar{X}$, and $y \in \bar{Y}$. We say that the set $X$ at $x$ is equivalent to the set $Y$ at $y$ if there exist $u \in \mathfrak{U}_{x}$ and $v \in \mathfrak{U}_{y}$ such that $(X \cap u)-x=(Y \cap v)-y$.

Suppose that $X, Y \in \Sigma$. For the algebra $\mathscr{A}_{Y}$ we write $p^{\prime}$ instead of $p$ and $q^{\prime}$ instead of $q$. If $x$, $y, u$, and $v$ are as in the above definition then we consider the mappings $\varphi: u \cap \bar{X} \rightarrow v \cap \bar{Y}$ and $\Phi_{u v}: p(u) \mathscr{A}_{X} p(u) \rightarrow p^{\prime}(v) \mathscr{A}_{Y} p^{\prime}(v):$

$$
\begin{gathered}
\varphi(z)=z+y-x, \quad z \in u \cap \bar{X} ; \\
\Phi_{u v}(a)=\left\{T_{\tau(y-x)} P_{\tau u} A_{\tau} P_{\tau u} T_{\tau(x-y)}\right\}_{\tau>0}+\mathscr{J}_{Y},
\end{gathered}
$$

where $a=\left\{\mathscr{A}_{\tau}\right\}_{\tau>0}+\mathscr{J}_{X} \in p(u) \mathscr{A}_{X} p(u)$. It is clear that the definition of $\Phi_{u v}(a)$ is independent of the choice of $\left\{A_{\tau}\right\}_{\tau>0}$. Obviously, $\Phi_{u v}$ is an isometric isomorphism of $p(u) \mathscr{A}_{X} p(u)$ onto $p^{\prime}(v) \mathscr{A}_{Y} p^{\prime}(v)$; moreover, $\Phi_{u v}(p(w))=p^{\prime}(\varphi(w))$ for all $w \in \mathfrak{U}_{x}$.

Suppose that $X, Y \in \Sigma$ and the set $X$ at $x$ is locally equivalent to the set $Y$ at $y$.

Define the relation $R_{x y} \subset \mathscr{A}_{X}^{\prime} \times \mathscr{A}_{Y}^{\prime}$, by putting $a R_{x y} b$ if there exist $u \in \mathfrak{U}_{x}$ and $v \in \mathfrak{U}_{y}$ such that $(X \cap u)-x=(Y \cap v)-y$ and $\Phi_{u v}(p(u) a p(u)) \stackrel{y}{\sim} p^{\prime}(v) b p^{\prime}(v)$.

Observe the following obvious properties of the relation $R_{x y}$ :

(a) if $a \in \mathscr{A}_{X}^{\prime}, b \in \mathscr{A}_{Y}^{\prime}$, and $a R_{x y} b$ then $q(a, x)=q^{\prime}(b, y)$;

(b) if $a_{1}, a_{2} \in \mathscr{A}_{X}^{\prime}, b_{1}, b_{2} \in \mathscr{A}_{Y}^{\prime}, a_{1} R_{x y} a_{2}, b_{1} R_{x y} b_{2}$, and $\lambda_{1}, \lambda_{2} \in \mathbb{C}$ then

$$
\left(\lambda_{1} a_{1}+\lambda_{2} a_{2}\right) R_{x y}\left(\lambda_{1} b_{1}+\lambda_{2} b_{2}\right), \quad a_{1} a_{2} R_{x y} b_{1} b_{2} .
$$

Proposition 5.1. Suppose that $X, Y \in \Sigma, x \in \bar{X}, y \in \bar{Y}$, and the set $X$ at $x$ is equivalent to the set $Y$ at $y$. Then there is a unique isomorphism $\operatorname{loc}_{x y}:\left(\mathscr{A}_{X}^{\prime}\right)_{x} \rightarrow\left(\mathscr{A}_{Y}^{\prime}\right)_{y}$ such that the relation $\operatorname{loc}_{x y}\left(\pi_{x}(a)\right)=\pi_{y}(b)$ is equivalent to the relation $a R_{x y} b$ for arbitrary $a \in \mathscr{A}_{X}^{\prime}$ and $b \in \mathscr{A}_{Y}^{\prime}$.

Proof. 1. Let $\widetilde{R}_{x y} \subset\left(\mathscr{A}_{X}^{\prime}\right)_{x} \times\left(\mathscr{A}_{Y}^{\prime}\right)_{y}$ be the relation defined by the rule

$$
\pi_{x}(a) \widetilde{R}_{x y} \pi_{y}(b) \Leftrightarrow a R_{x y} b, \quad a \in \mathscr{A}_{X}^{\prime}, b \in \mathscr{A}_{Y}^{\prime} .
$$

2. For each $a \in \mathscr{A}_{X}^{\prime}$, there is an element $b \in \mathscr{A}_{Y}^{\prime}$ such that $a R_{x y} b$; i.e., $\pi_{x}(a) \widetilde{R}_{x y} \pi_{y}(b)$. Indeed, suppose that $u \in \mathfrak{U}_{x}$ and $v \in \mathfrak{U}_{y}$ are such that $(u \cap X)-x=(v \cap Y)-y$. Then $b=\Phi_{u v}(p(u) a b(u))$ is the sought element.

3. Similarly, we can prove that, for every $b \in \mathscr{A}_{Y}^{\prime}$, there is an element $a \in \mathscr{A}_{X}^{\prime}$ such that $\pi_{x}(a) \widetilde{R}_{x y} \pi_{y}(b)$.

4. It follows from the properties (a) and (b) of the relation $R_{x y}$ and Sections 2 and 3 that $\widetilde{R}_{x y}$ is the graph of some isometric isomorphism which we denote by $\operatorname{loc}_{x y}$. The existence is proven.

5. If the morphism $\operatorname{loc}_{x y}$ satisfies the conditions of the proposition then its graph coincides obviously with $\widetilde{R}_{x y}$. The uniqueness is proven.

Proposition 5.2. Suppose that $X, Y \in \Sigma, x \in \bar{X}, y \in \bar{Y}$, the set $X$ at $x$ is equivalent to the set $Y$ at $y$, and $A \in W_{p}$. Then $j_{X}(A) R_{x y} j_{Y}(A)$; i.e.,

$$
\operatorname{loc}_{x y}\left(\pi_{x}\left(j_{X}(A)\right)\right)=\pi_{y}\left(j_{Y}(A)\right) .
$$

Proof. Suppose that $u \in \mathfrak{U}_{x}, v \in \mathfrak{U}_{y}$, and $(X \cap u)-x=(Y \cap v)-y$. Using the invariance of $A$ under translations, we obtain

$$
T_{\tau(y-x)} P_{\tau u} P_{\tau X} A P_{\tau X} P_{\tau u} T_{\tau(x-y)}=P_{\tau(v \cap Y)} A P_{\tau(v \cap Y)}
$$

for every $\tau>0$. Hence, $\Phi_{u v}\left(j_{X}(A)\right)=p(v) j_{Y}(A) p(v) \stackrel{y}{\sim} j_{Y}(A)$ and $j_{X}(A) R_{x y} j_{Y}(A)$. 
Proposition 5.3. Suppose that $M \in \Sigma, x \in \bar{M}, K$ is a cone with vertex 0 , and the set $M$ at $x$ is locally equivalent to the cone $K$ at 0 . Then there is a unique morphism $\theta_{x}: \mathscr{W}_{M} \rightarrow \operatorname{End}\left(P_{K_{x}} L_{p}\right)$ of Banach algebras such that $\theta_{x}\left(j_{M}(A)\right)=P_{K} A P_{K}$ for every $A \in W_{p}$. Moreover, $\theta_{x}\left(\mathscr{W}_{M}\right) \subset \mathscr{C}_{K}$, the relations

$$
\left\|\theta_{x}(a)\right\|=q(a, x) \leq|a|
$$

hold for every $a \in \mathscr{W}_{M}$, and the invertibility of $\theta_{x}(a)$ in $\operatorname{End}\left(P_{K} L_{p}\right)$ is equivalent to the invertibility of the element $\pi_{x}(a)$ in the quotient algebra $\left(\mathscr{W}_{M}\right)_{x}$.

Proof. Define the morphism $\theta_{x}$ by the formula

$$
\theta_{x}(a)=\operatorname{isocon}\left(\operatorname{loc}_{x 0}\left(\pi_{x}(a)\right)\right), \quad a \in \mathscr{W}_{K} .
$$

It follows from Propositions 4.1 and 5.2 that $\theta_{x}$ possesses the above properties. The uniqueness ensues from Proposition 1.2.

From (5.1) and Proposition 4.2 we obtain an explicit description for the morphism $\theta_{x}$ in terms of pointwise limit of operators.

Proposition 5.4. Suppose that $M \in \Sigma, x \in \bar{M}, K$ is a cone with vertex $0, u \in \mathfrak{U}_{0},(M-x) \cap u=$ $K \cap u$, and $a=\left\{A_{\tau}\right\}_{\tau>0}+\mathscr{J}_{M}$. Then

$$
\theta_{x}(a)=\underset{\tau \rightarrow+\infty}{\mathrm{s}-\lim _{\tau u}} P_{\tau} T_{-\tau x} A_{\tau} T_{\tau x} P_{\tau u}=\underset{\tau \rightarrow+\infty}{\mathrm{s}-\lim _{\tau x}} T_{\tau} T_{\tau x}
$$

and consequently

$$
\left\|\theta_{x}(a)\right\| \leq \liminf _{\tau \rightarrow+\infty}\left\|A_{\tau}\right\|
$$

Now, we need an assertion on "domination" of one point over another.

Proposition 5.5. Suppose that $M \in \Sigma, x, y \in \bar{M}, u \in \mathfrak{U}_{x}, v \in \mathfrak{U}_{0}, K$ is a cone with vertex 0 , $(M \cap u)-x=K \cap v$, and $y \in u$. Then the inequality $q(a, x) \geq q(a, y)$ holds for every $a \in \mathscr{A}_{M}^{\prime}$ and the invertibility of $\pi_{x}(a)$ in $\left(\mathscr{A}_{M}^{\prime}\right)_{x}$ implies the invertibility of $\pi_{y}(a)$ in $\left(\mathscr{A}_{M}^{\prime}\right)_{y}$.

Proof. Define the mapping $\varphi: u \rightarrow v$ by the formula $\varphi(z)=z-x$. Then the corresponding mapping $\Phi_{u v}$ establishes an isomorphism of the algebra $p(u) \mathscr{A}_{X}^{\prime} p(u)$ onto the algebra $p(v) \mathscr{A}_{K}^{\prime} p(v)$. Take an arbitrary $a \in \mathscr{A}_{X}^{\prime}$. It follows from Proposition 5.1 and Lemmas 4.2 and 4.1 that the invertibility of the element $p(u) \operatorname{ap}(u)$ in the algebra $p(u) \mathscr{A}_{X}^{\prime} p(u)$ is equivalent to the invertibility of $\pi_{x}(a)$ in $\left(\mathscr{A}_{X}^{\prime}\right)_{x}$, and $q(a, x)=|p(u) \operatorname{ap}(u)|$. On the other hand, since $u$ is a neighborhood of $y$, the invertibility of $p(u) \operatorname{ap}(u)$ in $p(u) \mathscr{A}_{X}^{\prime} p(u)$ implies the invertibility of $\pi_{y}(a)$; moreover, $q(a, y) \leq|p(u) a p(u)|$.

\section{$\S 6$. The Main Theorem}

In this section we suppose that $M$ is a polyhedron in $\mathbb{R}^{n}$ and $E$ is the set of its vertices.

Lemma 6.1. Let $a \in \mathscr{A}_{M}^{\prime}$. Then the invertibility of $a$ is equivalent to the fact that, for every point $x \in E$, the element $\pi_{x}(a)$ is invertible in $\left(\mathscr{A}_{M}^{\prime}\right)_{x}$. Moreover, $|a|=\max _{x \in E} q(a, x)$.

Proof. A similar assertion with $E$ replaced by $M$ ensues from Proposition 3.1 and the general Theorems 2.2 and 2.3 of the local method. Passage from $M$ to $E$ follows from Proposition 5.5, since for every point $y \in M$ we can obviously find a vertex $x \in E$ which "dominates" $y$ in the sense of Proposition 5.5. The lemma is proven.

In the product $\prod_{x \in E} \operatorname{End}\left(P_{K_{x}} L_{p}^{m}\right)$, consider the closed pure subalgebra $\widetilde{\mathscr{C}}$ generated by the families of the form $\left\{P_{K_{x}} A P_{K_{x}}\right\}_{x \in E}$, where $A \in W_{p}$. Clearly,

$$
\tilde{\mathscr{C}} \subset \prod_{x \in E} \mathscr{C}_{K_{x}}
$$


Theorem 6.1. There is a unique morphism

$$
\Theta: \mathscr{W}_{M} \rightarrow \prod_{x \in E} \operatorname{End}\left(P_{K_{x}} L_{p}\right)
$$

of Banach algebras such that $\Theta\left(j_{M}(A)\right)=\left\{P_{K_{x}} A P_{K_{x}}\right\}_{x \in E}$ for all $A \in W_{p}$. This morphism $\Theta$ is an isometry which agrees with inversion, has the image $\widetilde{\mathscr{C}}$, and is the product of the family of the morphisms $\left\{\theta_{x}\right\}_{x \in E}$.

Proof. Put $\Theta=\prod_{x \in E} \theta_{x}$. Then it follows from Proposition 5.3 and Lemma 6.1 that $\Theta$ is an isometry which agrees with inversion. By Proposition 1.1, $\Theta\left(\mathscr{W}_{M}\right)=\widetilde{\mathscr{C}}_{M}$. The uniqueness of $\Theta$ ensues from the relation $\mathscr{W}_{M}=\left[j_{M}\left(W_{p}\right)\right]$ and Proposition 1.2.

REMARK. In the one-dimensional case $(n=1$ and $M=[0,1])$ an assertion similar to Theorem 6.1 was proven in [6] by a constructive description of the algebras $\mathscr{W}_{M}$ and $\mathscr{C}_{K}$.

Corollary 6.1. Suppose that an element $a \in \mathscr{W}_{M}$ has representation $a=\left\{A_{\tau}\right\}_{\tau>0}+\mathscr{J}_{M}$. Then

$$
\lim _{\tau \rightarrow+\infty}\left\|A_{\tau}\right\|=|a|=\max _{x \in E}\left\|\theta_{x}(a)\right\|
$$

Proof. The corollary follows from Theorem 6.1 and (5.2).

Corollary 6.2. Suppose that $a \in \mathscr{W}_{M}$ and $a=\left\{A_{\tau}\right\}_{\tau>0}+\mathscr{J}_{M}$. Then the following conditions are equivalent:

(a) the element $a$ is invertible in $\mathscr{A}_{M}$;

(b) $\lim \sup _{\tau \rightarrow+\infty}\left\|\left(A_{\tau}\right)_{\tau M}^{-1}\right\|<+\infty$; i.e., there is $\tau_{0}>0$ such that the operators $A_{\tau}$ are invertible on $\tau M$ for $\tau>\tau_{0}$ and $\sup _{\tau>\tau_{0}}\left\|\left(A_{\tau}\right)_{\tau M}^{-1}\right\|<+\infty$;

(c) for each point $x \in E$ the operator $\theta_{x}(a)$ is invertible on $K_{x}$.

If these conditions are satisfied then

$$
\theta_{x}(a)_{K_{x}}^{-1}=\underset{\tau \rightarrow+\infty}{\mathrm{s}-\lim _{\tau \rightarrow}} T_{-\tau x}\left(A_{\tau}\right)_{\tau M}^{-1} T_{\tau x}
$$

for every point $x \in E$. Moreover,

$$
\lim _{\tau \rightarrow+\infty}\left\|\left(A_{\tau}\right)_{\tau M}^{-1}\right\|=\left|a^{-1}\right|=\max _{x \in E}\left\|\theta_{x}(a)_{K_{x}}^{-1}\right\| .
$$

In particular, for $a=j_{M}(A)$ and $A \in W_{p}$ we obtain

Corollary 6.3. Let $A \in W_{p}$. Then the following conditions are equivalent:

(a) the element $j_{M}(A)$ is invertible in $\mathscr{A}_{M}$;

(b) $\lim \sup _{\tau \rightarrow+\infty}\left\|A_{\tau M}^{-1}\right\|<+\infty$;

(c) the operator $A$ is invertible on $K_{x}$ for all $x \in E$.

If these conditions are satisfied then

$$
A_{K_{x}}^{-1}=\operatorname{s-lim}_{\tau \rightarrow+\infty} T_{-\tau x} A_{\tau M}^{-1} T_{\tau x}
$$

for every point $x \in E$. Moreover,

$$
\lim _{\tau \rightarrow+\infty}\left\|A_{\tau M}^{-1}\right\|=\max _{x \in E}\left\|A_{K_{x}}^{-1}\right\| .
$$

Relation (6.1) was stated in the introduction as Proposition 0.1. The other assertions of Corollary 6.3 were proven by A. V. Kozak in [13-15]. He also proved similar results for weak and composite convolutions and for sets $M$ of a more general form than polyhedra. 


\section{$\S$ 7. Limits of Pseudospectra}

In this section, from Corollary 6.2 we derive the theorem on the limit of pseudospectra. The idea of the proof is borrowed from [6].

First, we give the necessary information about the norm of the resolvent, pseudospectrum, and the Hausdorff metric.

Let $\mathscr{A}$ be a Banach algebra (with unit $e$ and norm $|\cdot|$ ) and let $\dot{\mathbb{C}}=\mathbb{C} \cup\{\infty\}$ be the extended complex plane. Define the function $\mathrm{nr}: \mathscr{A} \times \dot{\mathbb{C}} \rightarrow[0,+\infty]$ (the "norm of the resolvent") by the formula

$$
\operatorname{nr}(a, \lambda)= \begin{cases}\left|(\lambda e-a)^{-1}\right|, & \lambda \in \mathbb{C} \backslash \sigma(a), \\ +\infty, & \lambda \in \sigma(a), \\ 0, & \lambda=\infty .\end{cases}
$$

It is easy to see that the function $\operatorname{nr}(a, \cdot)$ is continuous on $\dot{\mathbb{C}}$. In particular,

$$
|\operatorname{nr}(a, \mu)-\operatorname{nr}(a, \lambda)|<\frac{|\mu-\lambda| \operatorname{nr}(a, \lambda)^{2}}{1-|\mu-\lambda| \operatorname{nr}(a, \lambda)} .
$$

Theorem 7.1. Suppose that $(\Omega, \mu)$ is a measure space, $p \in(1,+\infty), \mathscr{A}=\operatorname{End}\left(L_{p}(\Omega, \mu)\right)$, and $a \in \mathscr{A}$. Then the maximum principle holds for the norm of the resolvent of the element a; i.e., the function $\operatorname{nr}(a, \cdot)$ has no local maximum on $\mathbb{C} \backslash \sigma(a)$.

This theorem was proven in [6]. It is clear that we can replace $\operatorname{End}\left(L_{p}(\Omega, \mu)\right)$ in Theorem 7.1 with $\operatorname{End}\left(L_{p}^{m}(\Omega, \mu)\right)$. The maximum principle for the norm may fail for arbitrary operator-valued analytic functions (see a counterexample in $[6]$ ).

If $a \in \mathscr{A}$ and $\varepsilon>0$ then the $\varepsilon$-pseudospectrum of $a$ is defined by the equality

$$
\sigma_{\varepsilon}(a)=\{\lambda \in \mathbb{C} \mid \operatorname{nr}(a, \lambda) \geq 1 / \varepsilon\} .
$$

Clearly, $\sigma_{\varepsilon}(a)$ is a compact set in $\mathbb{C}$; moreover, $\sigma(a) \subset \sigma_{\varepsilon}(a)$.

Recall the definition of the Hausdorff metric $d_{H}$. Let $\mathscr{K}$ be a collection of nonempty compact sets in $\mathbb{C}$. For arbitrary $X, Y \in \mathscr{K}$

$$
d_{H}(X, Y)=\max \left(\sup _{x \in X} \inf _{y \in Y}|x-y|, \sup _{y \in Y} \inf _{x \in X}|x-y|\right) .
$$

Thus, the inequality $d_{H}(X, Y)<\delta$ means that $X \subset U(Y, \delta)$ and $Y \subset U(X, \delta)$, where

$$
U(X, \delta)=\left\{z \in \mathbb{C}\left|\inf _{x \in X}\right| z-x \mid<\delta\right\}
$$

It is well known that $\left(\mathscr{K}, d_{H}\right)$ is a complete metric space. If $\left\{X_{\tau}\right\}_{\tau>0}$ is a directed family in $\mathscr{K}$ and $Y \in \mathscr{K}$ then the notations $X_{\tau} \rightarrow Y$ and $\lim _{\tau} X_{\tau}=Y$ mean that $d_{H}\left(X_{\tau}, Y\right) \rightarrow 0$ which is equivalent to the following: for every $\delta>0$, there is $\tau_{0}>0$ such that $X_{\tau} \subset U(Y, \delta)$ and $Y \subset U\left(X_{\tau}, \delta\right)$ for $\tau>\tau_{0}$.

As in $\S 6$, let $M$ be a polyhedron in $\mathbb{R}^{n}$ and let $E$ be the set of its vertices. In the proof of the following theorem, it is essential that $E$ is finite. We additionally suppose that $p \in(1,+\infty)$. This is necessary for the applicability of Theorem 7.1.

Theorem 7.2. Suppose that $a \in \mathscr{W}_{M}, a=\left\{A_{\tau}\right\}_{\tau>0}+\mathscr{J}_{M}$, and $\varepsilon>0$. Then

$$
\lim _{\tau \rightarrow+\infty} \sigma_{\varepsilon}\left(A_{\tau}\right)=\sigma_{\varepsilon}(a)=\sigma_{\varepsilon}(\Theta(a))=\bigcup_{x \in E} \sigma_{\varepsilon}\left(\theta_{x}(a)\right) .
$$


Proof. 1. From Corollary 6.2 to Theorem 6.1 we obtain

$$
\sigma_{\varepsilon}(a)=\sigma_{\varepsilon}(\Theta(a))=\bigcup_{x \in E} \sigma_{\varepsilon}\left(\theta_{x}(a)\right) .
$$

We are left with proving that $\sigma_{\varepsilon}\left(A_{\tau}\right) \rightarrow \sigma_{\varepsilon}(a)$ as $\tau \rightarrow+\infty$.

2. Prove that, for every $\delta>0$, there is $\tau_{0}>0$ such that $\sigma_{\varepsilon}\left(A_{\tau}\right) \subset U\left(\sigma_{\varepsilon}(a), \delta\right)$ for $\tau>\tau_{0}$. For each point $\lambda$ of the compact set $Q=\dot{\mathbb{C}} \backslash U\left(\sigma_{\varepsilon}(a), \delta\right)$ we have

$$
\lim _{\tau \rightarrow+\infty} \operatorname{nr}\left(A_{\tau}, \lambda\right)=\operatorname{nr}(a, \lambda)=1 / \varepsilon-\xi_{\lambda}
$$

where $\xi_{\lambda}>0$. Hence, we find $\tau_{\lambda}>0$ such that $\operatorname{nr}\left(A_{\tau}, \lambda\right)<1 / \varepsilon-\xi_{\lambda} / 2$ for $\tau>\tau_{\lambda}$. Using (7.1), we find a neighborhood $U_{\lambda}$ of $\lambda$ such that $\operatorname{nr}\left(A_{\tau}, \mu\right)<1 / \varepsilon-\xi_{\lambda} / 3$ for all $\mu \in U_{\lambda}$ and $\tau>\tau_{\lambda}$. The family $\left\{U_{\lambda}\right\}_{\lambda \in S}$ constitutes an open covering of the compact set $Q$. Extract a finite set $\Lambda \subset Q$ such that the family $\left\{U_{\lambda}\right\}_{\lambda \in \Lambda}$ covers $Q$ and put $\tau_{0}=\max \left\{\tau_{\lambda}: \lambda \in \Lambda\right\}$ and $\xi=\min \left\{\xi_{\lambda}: \lambda \in \Lambda\right\}$. Then

$$
\operatorname{nr}\left(A_{\tau}, \mu\right)<1 / \varepsilon-\xi / 3<1 / \varepsilon
$$

for $\tau>\tau_{0}$ and $\mu \in Q$. We have thus proven that $\sigma_{\varepsilon}\left(A_{\tau}\right) \subset \mathbb{C} \backslash Q=U\left(\sigma_{\varepsilon}(a), \delta\right)$ for $\tau>\tau_{0}$.

3. Take arbitrary $\delta>0$ and $x \in E$, put $B_{x}=\theta_{x}(a)$, and prove the existence of $\tau_{x}^{\prime}>0$ such that $\sigma_{\varepsilon}\left(B_{x}\right) \subset U\left(\sigma_{\varepsilon}\left(A_{\tau}\right), \delta\right)$ for $\tau>\tau_{x}^{\prime}$. For each point $\lambda \in \sigma_{\varepsilon}\left(B_{x}\right)$, consider its $\delta / 2$-neighborhood $U(\lambda, \delta / 2)$. Using Theorem 7.1 (on the norm of the resolvent), find $\mu \in U(\lambda, \delta / 2)$ such that $\operatorname{nr}\left(B_{x}, \mu\right)>1 / \varepsilon$. Now, using the relation

$$
\lim _{\tau \rightarrow+\infty} \operatorname{nr}\left(A_{\tau}, \mu\right)=\operatorname{nr}(a, \mu) \geq \operatorname{nr}\left(B_{x}, \mu\right),
$$

take $\tau_{\lambda}^{\prime}>0$ such that $\operatorname{nr}\left(A_{\tau}, \mu\right)>1 / \varepsilon$ for $\tau>\tau_{\lambda}^{\prime}$. For $\tau>\tau_{\lambda}^{\prime}$ we then have $\mu \in \sigma_{\varepsilon}\left(A_{\tau}\right), \lambda \in U\left(\sigma_{\varepsilon}\left(A_{\tau}\right)\right.$, $\delta / 2)$, and $U(\lambda, \delta / 2) \subset U\left(\sigma_{\varepsilon}\left(A_{\tau}\right), \delta\right)$. Extracting a finite subcovering from the covering $\{U(\lambda, \delta / 2): \lambda \in$ $\left.\sigma_{\varepsilon}\left(B_{x}\right)\right\}$ of the compact set $\sigma_{\varepsilon}\left(B_{x}\right)$, we find $\tau_{x}^{\prime}>0$ such that $\sigma_{\varepsilon}\left(B_{x}\right) \subset U\left(\sigma_{\varepsilon}\left(A_{\tau}\right), \delta\right)$ for $\tau>\tau_{x}^{\prime}$.

4. Now, take an arbitrary $\delta>0$, for each point $x \in E$ construct $\tau_{x}^{\prime}$ as in Section 3 , and put $\tau^{\prime}=\max _{x \in E} \tau_{x}^{\prime}$. Then

$$
\sigma_{\varepsilon}(a)=\bigcup_{x \in E} \sigma_{\varepsilon}\left(B_{x}\right) \subset U\left(\sigma_{\varepsilon}\left(A_{\tau}\right), \delta\right)
$$

for $\tau>\tau^{\prime}$. This, together with Section 2, implies that

$$
\lim _{\tau} \sigma_{\varepsilon}\left(A_{\tau}\right)=\sigma_{\varepsilon}(a)
$$

The theorem is proven.

Proposition 0.2 is obtained from Theorem 7.2 for $a=j_{M}(A)$.

\section{References}

1. Landau H., "The notion of approximate eigenvalues applied to an integral equation of laser theory," Quart. Appl. Math., 35, 165-171 (April 1977).

2. Reichel L. and Trefethen L. N., "Eigenvalues and pseudo-eigenvalues of Toeplitz matrices," Linear Algebra Appl., 162, 153-185 (1992).

3. Böttcher A., "Pseudospectra and singular values of large convolution operators," J. Integral Equations Appl., 6, 267-301 (1994).

4. Böttcher A. and Wolf H., "Spectral approximation for Segal-Bargmann space Toeplitz operators," Linear Operators, Banach Center Publ (Warsaw), 387, 25-48 (1997).

5. Grudsky S. M. and Kozak A. V., "On the convergence rate for norms of operators inverse to truncated Toeplitz operators," in: Integrodifferential Equations and Some of Their Applications [in Russian], Rostov Univ., Rostov-on-Don, 1995 , pp. $45-55$.

6. Böttcher A., Grudsky S. M., and Silbermann B., "Norms of inverses, spectra, and pseudospectra of large truncated Wiener-Hopf operators and Toeplitz matrices," New York J. Math., 3, 1-31 (1997).

7. Böttcher A. and Silbermann B., Introduction to Large Truncated Toeplitz Matrices, Springer-Verlag, New York (1999). 
8. Simonenko I. B., "A new general method for studying linear operator equations analogous to singular integral equations," Dokl. Akad. Nauk SSSR, 158, 790-793 (1964).

9. Simonenko I. B., "A new general method for studying linear operator equations analogous to singular integral equations. I," Izv. Akad. Nauk SSSR Ser. Mat., 29, 567-586 (1965).

10. Simonenko I. B., "Convolution operators in cones," Mat. Sb., 74, 298-313 (1967).

11. Simonenko I. B. and Chin' Ngok Min', A Local Method in the Theory of One-Dimensional Singular Integral Equations with Piecewise Continuous Coefficients. Noethericity [in Russian], Rostov Univ., Rostov-on-Don (1986).

12. Simonenko I. B., "The theory of local-type operators and some of their applications," submitted to VINITI on January 23, 1996, No. 275-B96.

13. Kozak A. V., "The local principle in the theory of projection methods," Dokl. Akad. Nauk SSSR, 212, 1287-1289 (1973).

14. Kozak A. V., "On the reduction method for discrete multidimensional convolutions," Mat. Issled. (Kishinëv), 8, 157-160 (1973).

15. Kozak A. V., Projection Methods for Solving Multidimensional Equations of Convolution Type, Diss. Kand. Fiz.-Mat. Nauk, Rostov-on-Don, 1974.

16. Kozak A. V. and Simonenko I. B., "Projection methods for solving multidimensional discrete equations in convolutions," Sibirsk. Mat. Zh., 21, 119-127 (1980).

17. Krupnik N. Ya., "Exact constant in Simonenko's theorem on the envelope of a family of operators of local type," Funktsion. Anal. i Prilozhen., 20, 70-71 (1986). 